\title{
A CHARACTERIZATION OF LIGHT OPEN MAPS OF EUCLIDEAN SPACES INTO EUCLIDEAN SPACES ${ }^{1}$
}

\section{JAMES J. ANDREWS}

We will use the term open map to mean a map $f$ of $X$ into $Y$ such that $f(U)$ is open in $Y$ for every open set $U$ in $X$. A map $f$ of $R^{n}$ (Euclidean $n$-space) into $R^{m}$ is pseudo-monotone if and only if $R^{n}-f^{-1}(X)$ has no bounded component for every closed set $X$ in $R^{n}$ such that $R^{m}-X$ has no bounded component.

The purpose of this note is to prove that a light map $f$ of $R^{n}$ into $R^{m}$ is open if and only if $f$ is pseudo-monotone. But first we must prove the following lemma:

LEMma. If $f$ is an open map of $R^{n}$ into $R^{m}, U$ is a bounded open set in $R^{n}$, and $f(U) \cap W \neq \varnothing$ for some component $W$ of $R^{m}-f$ (bdry $U$ ), then $W \subset f(U)$. Thus, in particular, $f(U)$ does not intersect the unbounded component of $R^{m}-f$ (bdry $U$ ).

Proof. Assume $W \nsubseteq f(U)$. Let $p$ be a point of $W-f(U)$ and $q$ be a point of $W \cap f(U)$. Let $\bar{p} \bar{q}$ be an arc from $p$ to $q$ in $R^{n}-f$ (bdry $U$ ). Since $q$ is in the compact set $f(\bar{U})$ and $p$ is not, there is a first point $x$ on the arc $\bar{q} \bar{q}$ in the order $p$ to $q$ such that $x$ is in $f(\bar{U})$. We note $x$ is not in $f$ (bdry $U$ ) since $\bar{p} \bar{q} \cap f$ (bdry $U)=\varnothing$. Therefore $f^{-1}(x) \cap U \neq \varnothing$ and $x \subset$ bdry $f(U)$. Hence $f$ is not open.

REMARK. If $W$ is the union of $X$ and the bounded components of $R^{m}-X$, then $R^{m}-W$ has no bounded components.

Theorem. A light map $f$ of $R^{n}$ into $R^{m}$ is open if and only if $f$ is pseudo-monotone.

Proof. Assume $f$ is open but not pseudo-monotone. Then by definition of pseudo-monotone there is a closed set $X$ in $R^{m}$ such that $R^{m}-X$ has no bounded component and $R^{n}-f^{-1}(X)$ has a bounded component. Let $R^{n}-f^{-1}(X)=A \cup B$ where $A$ and $B$ are open, $A \cap B$ $=\varnothing$, and $A$ is both bounded and nonempty. For the open set $A$ we have $f$ (bdry $A) \subset X$ and $f(A) \subset R^{m}-X$. Hence $f(A) \cap W \neq \varnothing$ for some unbounded component $W$ of $R^{m}-X$. $\left(R^{m}-X\right.$ has no bounded component.) But $R^{m}-X \subset R^{m}-f$ (bdry $A$ ). Therefore $f(A) \cap W^{\prime} \neq \varnothing$,

Presented to the Society, April 6, 1957 under the title $A$ characteristic of light open maps of locally Euclidean spaces into locally Euclidean spaces; received by the editors March 22, 1957 and, in revised form, April 18, 1958.

1 This research was supported by National Science Grant NSF-G3016. 
where $W^{\prime}$ is the unbounded component of $R^{m}-f$ (bdry $A$ ) containing $W$. By our lemma this is not possible.

Assume $f$ is pseudo-monotone but not open. Then there is an open set $U$ in $R^{n}$ such that $f(U)$ is not open in $R^{m}$. Let $p$ be a point of $f(U) \cap$ bdry $f(U)$, let $q$ be a point of $f^{-1}(p) \cap U$, and let $V$ be a bounded neighborhood of $q$ lying in $U$ such that the boundary of $V$ does not intersect the 0 -dimensional set $f^{-1}(p)$. The boundary of $V=B$ is compact, hence there is a spherical neighborhood $S$ of $p$ which does not intersect the compact set $f(B)$. Let $x$ be a point of the set $S-f(U)$. $S-f(U)$ is not empty because $p$ is the boundary of $f(U)$. Let $b$ be the first point on the line segment from $x$ to $p$ which is in the compact set $f(\bar{V})$. Then $b$ is in $f(V)$ since $b$ is in $S$ and $S \cap f$ (bdry $V)=\varnothing$. Let $a$ be a point of $f^{-1}(b) \cap V$. Let $r$ denote the ray from $p$ through $x$ and let $r^{\prime}$ denote the subray of $r$ with source at $x$. Let $S^{\prime}$ denote the open set $S-r^{\prime}$. Let $V^{\prime}$ be a neighborhood of $a$, whose closure lies in the open set $f^{-1}(S) \cap V$, and whose boundary does not intersect the 0 dimensional set $f^{-1}(b) . b$ is in $f\left(V^{\prime}\right) \cap r$ and is the only point of the ray $r^{\prime} \cup \bar{x} \bar{b}$ which lies in $f\left(\bar{V}^{\prime}\right)$. Hence $b$ is in an unbounded component of $R^{m}-f$ (bdry $V^{\prime}$ ). Let $W$ be the union of $f$ (bdry $V^{\prime}$ ) and the bounded components of $f$ (bdry $V^{\prime}$ ), then $R^{m}-W$ has no bounded components. Consider the set $f^{-1}(W) . a \notin f^{-1}(W)$ because $b \notin W$. But bdry $V^{\prime}$ $\subset f^{-1}(W)$; hence the bounded set $V^{\prime} \subset f^{-1}(W)$ and therefore $a \in f^{-1}(W)$.

In showing that an open map is pseudo-monotone we did not use the fact that $f$ was light. Hence an open map of $R^{n}$ into $R^{m}$ is pseudomonotone.

In comparing the theorem with a result of A. D. Wallace [2], the referee points out that the following proposition is false:

Proposition. If $f$ is a pseudo-monotone map of $R^{n}$ into $R^{m}$, then there exists an increasing sequence $C_{1}, C_{2}, \cdots$ of $n$-cells such that $U C_{i}=R^{n}$ and $f / C_{i}$ is quasi-monotone.

\section{BIBLIOGRAPHY}

1. W. Hurewicz and H. Wallman, Dimension theory, Princeton, 1941.

2. A. D. Wallace, Quasi-monotone transformations, Duke Math. J. vol. 7 (1940) pp. 136-145.

UNIVERSITY OF GEORGIA 